tion and the use of cholestyramine was associated with a significant reduction in adverse cardiac outcomes. Analysis of the LRC-CPPT findings suggests that each 1 percent reduction in total serum cholesterol results in a 2 percent reduction in the incidence of coronary heart disease rates.

In December 1984, the National Institutes of Health sponsored a consensus development conference to review the scientific evidence and to make public health and clinical practice recommendations on blood cholesterol reduction. The outgrowth of the conference was the establishment of the National Cholesterol Education Program. The osteopathic representative to the program is Dr. William Voss, a general internist from Jefferson City, Missouri. He commented, "As the AOA's representative, my job is to bring information back to our profession and bring our people into the federal program."

Several surveys have revealed that public awareness of the importance of cholesterol is very high. At the same time, these surveys indicate that physicians in this country are either unaware or unconvinced of the importance of measuring and managing serum cholesterol levels to prevent cardiovascular disease. Lesko and Abbey's paper, "Elevated serum cholesterol values preceding myocardial infarction: The need for physician referral to lipid education," which begins on page $36 / 75$ of this issue, is supportive of the survey results. In their study, the records of 66 patients with a discharge diagnosis of myocardial infarction were evaluated. Only 11 percent of the patients with a serum cholesterol level $\geqslant 240 \mathrm{mg}$./dl. were referred to a lipid education class prior to their myocardial infarction. The authors contend that myocardial infarctions may be prevented if physicians screen their patients for elevated serum cholesterol levels and make use of available lipid education classes.

The American Heart Association, in recognition of the need to improve physician education, will conduct a national physician training conference in the fall of 1987 . Local representatives of the 55 American Heart Association affiliates will receive instruction to become trainers. They will also be given a portable instruction kit so that a "ripple effect" of education may spread throughout the community of primary care physicians. It is my honor to have been invited to represent the osteopathic medical profession as a member of the American Heart Association Cholesterol Education Program Ad Hoc Advisory Task Force.

Physicians can take the first step toward increasing their awareness of cholesterol management by sending for the pamphlet, "Cholesterol counts: Steps for lowering your patient's blood cholesterol."
Write to the National Heart, Lung, and Blood Institute, Public Inquiries and Reports Branch, Building 32, Room 4A21, National Institutes of Health, Bethesda, MD 20205. Physicians may also wish to clip out and distribute to their patients the January "Patient Health Guide." "Lowering cholesterol levels for better health" explains the new cholesterol guidelines from the consensus conference and offers practical advice on trimming fat and cholesterol from the diet.

THOMAS W. ALLEN, D.O., FACOI

\section{AIDS-A major public health danger}

The public can no longer afford the commonly held misconception that AIDS is a disease only of homosexuals and intravenous drug users. Heterosexuality in itself offers no protection against infection with the AIDS-causing virus. According to U.S. Surgeon General C. Everett Koop, whose report appears in this issue of JAOA, "Although those practicing high risk behavior who become infected with the AIDS virus are found mainly among homosexual and bisexual men and male and female intravenous drug users, heterosexual transmission is expected to account for an increasing proportion of those who become infected with the AIDS virus in the future." He calls upon the public to abandon the feeling that "people from certain groups deserve their illness." He reminds us that we are fighting a disease, not people.

The dimensions of this disease are sobering. By the end of 1991 (just 10 years since the first report of AIDS in this country), an estimated 270,000 cases and 179,000 deaths will have occurred. In the year 1991 alone, an estimated 145,000 patients with AIDS will need health and supportive services, at a total cost of between $\$ 8$ and $\$ 16$ billion. In this same year, 54,000 deaths from AIDS are expected.

The American Osteopathic Association supports Dr. Koop in this effort. The AOA House of Delegates adopted the following resolution at its 1986 annual meeting:

RESOLVED, that the American Osteopathic Association encourage its membership to educate its patients, communities and peers on the known facts and fallacies of the Acquired Immunodeficiency Syndrome (AIDS); and be it further

RESOLVED, that the AOA stress that all osteopathic physicians are a fundamental part of the treatment team in AIDS. 
In this issue of JAOA, two original papers related to AIDS are found, along with the complete text of the surgeon general's report on AIDS. Dr. Koop's report, which was released on October 22, 1986, begins on page $64 / 103$.

"Radiologic manifestations of acquired immunodeficiency syndrome," by Pera, Weisgrau, Harle, and Rios, is an excellent and comprehensive review. The various radiologic presentations of the disease have been compiled according to the three body systems most affected by AIDS - the central nervous system, the gastrointestinal system, and the pulmonary system. The authors point out that radiologic manifestations are often the initial findings in AIDS. The report begins on page 48/87.

"Psychiatric aspects of AIDS: Overview for the general practitioner," by Johnson, appears on page 60/99. According to Dr. Johnson, it is not surprising that AIDS patients are at special risk for psychiatric illness, given the bleak prognosis, the complex pathophysiology, and the social implications of the disease. In addition to organic brain syndromes, opportunistic infections, and tumors, many patients have pre-existing illnesses that are exacerbated by the diagnosis of AIDS. Dr. Johnson calls for a holistic approach to treatment, and asks that physicians consider the patient's emotional as well as medical needs when applying osteopathic principles to the patient's total treatment plan.

Readers may wish to refer to the December issue of THE Do for additional information on the federal AIDS policy, as well as an in-depth look at how both physicians and patients are confronting the complexities of AIDS. The "Patient Health Guide" in the December issue of the JAOA also tackles the subject in its discussion of "The blood supply in the age of AIDS." This patient-education handout allays fears about blood transfusions by outlining the steps that have been taken to safeguard the blood supply.

THOMAS W. ALLEN, D.O., FACOI

\section{The importance of the negative test}

The January issue of THE Do features a series of information sheets that can be used for patient education. It explains in easy-to-understand terms what some of the more common clinical tests are about, and what to expect when they are performed. The general belief is that the more facts people have, the less they have to fear.

In this era of public and professional emphasis on the cost of medical care, tests that tend to produce negative results are being discouraged. It is dangerous, however, to subscribe to the philosophy that a negative test contributes nothing but cost to the rendering of quality medical care.

Those of us who have been in practice for any length of time soon realize that it is as important to the patient to know what is not wrong as it is to know what is wrong. For example, if a whole battery of tests are performed, a simple statement that "all your tests were negative" does not always allay a person's fears. It is just as easy to say that "your electrocardiogram did not reveal any heart disease, the blood test did not show any evidence of anemia, and the chest $x$-ray did not reveal any abnormality that might have serious consequences." The same approach applies to blood pressure readings. It is amazing how often a patient reports that his physician took his blood pressure but never told him whether it was high or low.

From the patient's standpoint and for the physician who is trying to make a differential diagnosis, the negative laboratory test or $\mathrm{x}$-ray is as important as a positive result. All findings should be conveyed to the patient. As an example, a patient complained of knee pain that had persisted for 2 or 3 months. Although the history and physical examination indicated some degree of chronic medial meniscus pathology, $x$-rays were ordered. They were negative, and the patient was notified. On the spur of the moment, the doctor added that there was no evidence of malignancy. Before the physician could retract what he considered an unnecessary and possibly alarming remark, the patient interjected, "Thank God! The pain had lasted so long-I was sure it was cancer."

The physician's office should be a place where diseases and malfunctions are discovered and treated. But it should also be a place where patients are reassured and given understanding of what is and what is not wrong.

In the practice of quality medicine, there is no basis for disparaging the importance of the negative finding. More importantly, there is no justification for not communicating negative results to a worried patient.

GEORGE W. NORTHUP, D.O., FAAO

\section{$\overline{\text { editorial comment }}$}

The relationship between trace metals and the normal aging process was examined at a recent conference, which was jointly sponsored by the continued on page 21/58 